\title{
Risk factors for acute kidney injury in elderly patients after coronary artery bypass grafting: A Chinese population
}

\author{
Xuejian $\mathrm{Hou}^{1}$, Taoshuai $\mathrm{Liu}^{1}$, shijun $\mathrm{xu}^{1}$, Zhuhui Huang ${ }^{1}$, Yang $\mathrm{Li}^{1}$, and Ran Dong ${ }^{1}$ \\ ${ }^{1}$ Affiliation not available
}

February 22, 2021

\begin{abstract}
Background and aim of the study: Elderly patients are more likely to have adverse complications after coronary artery bypass grafting (CABG). There are few studies on the risk factors for acute kidney injury (AKI) after surgery in elderly patients, especially in the Asian population. This study retrospectively analysed the risk factors for AKI in Chinese elderly patients after CABG and established a risk prediction model to detect these risk factors early and take active intervention measures. Methods: A total of 432 patients were included in this study from 2018 to 2019 . AKI was defined according to the Kidney Disease Improving Global Outcomes (KDIGO) criteria. The patients were divided into an AKI group and a non-AKI group. Multivariate logistic regression analysis was used to screen out the factors with $\mathrm{p}<0.05$. Results: Out of 432 patients in the study, 119 (27.5\%) developed AKI. The estimated glomerular filtration rate (eGFR), [?]3 coronary anastomoses, preoperative intra-aortic balloon pump (IABP) implantation and prolonged ventilation time were independent risk factors for AKI. The area under the ROC curve was 0.702 (95\% confidence interval (CI) [0.643-0.761], p < 0.001). Conclusions: The eGFR, [?]3 coronary anastomoses, preoperative IABP implantation and prolonged ventilation time are independent risk factors for AKI in elderly patients undergoing coronary artery bypass grafting. Early discovery of these risk factors and the implementation of appropriate intervention measures are useful to reduce the occurrence of AKI after CABG and improve the prognosis of patients.
\end{abstract}

Risk factors for acute kidney injury in elderly patients after coronary artery bypass grafting: A Chinese population

Xuejian Hou ${ }^{1}$ MD; Taoshuai Liu ${ }^{1}$ MD; Shijun Xu ${ }^{1}$ MD; Zhuhui Huang ${ }^{1}$ MD; Yang Li ${ }^{1}$ MD; Ran Dong MD 1

${ }^{1}$ Department of Cardiac Surgery, Beijing Anzhen Hospital, Capital Medical University, Beijing, China

Correspondence should be addressed to Ran Dongdrdongran@126.com and Yang Li li13488833585@163.com

Address: No.2 Anzhen Road, Chaoyang District, Beijing, P.R.China

Phone: 86-10-6445 6411

Running title: The AKI after CABG

Data availability statement: The datasets used and/or analyzed during the current study are available from the corresponding author on reasonable request.

Funding: This work was supported by funding of the Beijing Hospital Authority Project (PX2018027) and the National Natural Science Foundation of China (grant nos. 81570373 and 81770412).

Conflict of interest disclosure: There was no conflicts of interest to declare. 
Institutional Review Board approval or waive: The study was approved by the Ethics Committee of Beijing Anzhen Hospital (Approval Numbers: 2010043X).

Patients consent statement: The consent was obtained from the study participants oral.

Permission to reproduce material from other sources: There was no reproduce material.

Clinical trial registration: It is a retrospective study without clinical registration.

\section{Abstract}

Background and aim of the study: Elderly patients are more likely to have adverse complications after coronary artery bypass grafting (CABG). There are few studies on the risk factors for acute kidney injury (AKI) after surgery in elderly patients, especially in the Asian population. This study retrospectively analysed the risk factors for AKI in Chinese elderly patients after CABG and established a risk prediction model to detect these risk factors early and take active intervention measures.

Methods: A total of 432 patients were included in this study from 2018 to 2019. AKI was defined according to the Kidney Disease Improving Global Outcomes (KDIGO) criteria. The patients were divided into an AKI group and a non-AKI group. Multivariate logistic regression analysis was used to screen out the factors with $p<0.05$.

Results: Out of 432 patients in the study, 119 (27.5\%) developed AKI. The estimated glomerular filtration rate (eGFR), [?] 3 coronary anastomoses, preoperative intra-aortic balloon pump (IABP) implantation and prolonged ventilation time were independent risk factors for AKI. The area under the ROC curve was 0.702 (95\% confidence interval (CI) [0.643-0.761], $p<0.001$ ).

Conclusions: The eGFR, [?]3 coronary anastomoses, preoperative IABP implantation and prolonged ventilation time are independent risk factors for AKI in elderly patients undergoing coronary artery bypass grafting. Early discovery of these risk factors and the implementation of appropriate intervention measures are useful to reduce the occurrence of AKI after CABG and improve the prognosis of patients.

\section{Keywords: Acute kidney injury(AKI), Coronary artery bypass grafting (CABG)}

Introduction: The incidence of acute kidney injury after CABG is high. According to the definition of AKI, the incidence rate has been reported to range from $6.7 \%$ to $39 \%^{1-3}$. AKI is associated with increased complications and mortality after $\mathrm{CABG}^{3-5}$, which further increases in the more severe stage of AKI; acute kidney injury after cardiac surgery is independently associated with increased short-term and long-term mortality ${ }^{4,6-9}$. AKI after cardiac surgery also increases intensive care unit (ICU) length of stay and resource utilization $^{2,10}$.

The development of AKI involves a variety of mechanisms, including ischemic reperfusion injury, renal toxin release, haemolysis, oxidative stress and cytokine secretion, which can cause a systemic inflammatory response, endothelial damage and renal tubular cell damage ${ }^{1,11-13}$. Many previous studies have shown that older age, low ejection fraction, a previous history of kidney disease, and long-term cardiopulmonary bypass are important predictors of AKI development ${ }^{6,14-16}$.

With the popularization of CABG operations, an increasing number of patients with cardiovascular complications receive CABG treatment. Due to postoperative complications, the results may be unfavourable. Especially for the elderly patients, due to the relatively poor basic renal function of elderly patients, adverse complications after CABG are more likely to occur ${ }^{17}$. However, there are few studies on the risk factors for AKI after CABG in elderly patients, especially in the Asian population. Therefore, this study retrospectively analysed the risk factors for AKI in Chinese elderly patients after CABG and established a risk prediction model. Early detection of these risk factors and active intervention measures will help to reduce the occurrence of AKI after CABG and improve the prognosis of elderly patients.

Materials and Methods 


\section{Patients and Setting}

A total of 500 patients aged over 70 years who underwent CABG for only the first time from 2018 to 2019 were included in this study. Among them, 68 patients lacked clinical data, and a total of 432 patients were finally included. According to the KDIGO diagnostic criteria of $\mathrm{AKI}^{18}$, patients were divided into an AKI group and a non-AKI group. There were 119 patients in the AKI group and 313 patients in the non-AKI group.

\section{Medical Ethics}

The study was approved by the Ethics Committee of Beijing Anzhen Hospital (Approval Numbers: 2010043X). All consents were obtained from the study participants oral.

\section{Definition of AKI}

AKI was defined according to the Kidney Disease Improving Global Outcomes (KDIGO) criteria ${ }^{18}$ : an increase in serum creatinine (Scr [?] $0.3 \mathrm{mg} / \mathrm{dl}$ ) or an increase in Scr [?]1.5times baseline in 7 days after surgery or urine volume [?] $0.5 \mathrm{ml} / \mathrm{kg} / \mathrm{h}$ for $6 \mathrm{~h}$.

\section{Definition of Elderly}

Elderly referred to people over 70 years old.

\section{Data Collection}

Detailed clinical information: This information included age, sex, body mass index(BMI), symptom status (New York Heart Association (NYHA) classification), previous cardiac history (previous myocardial infarction and previous percutaneous coronary intervention (PCI)), diabetes, hypertension, hyperlipidaemia, peripheral vascular disease, previous stroke or chronic obstructive pulmonary disease, smoking, baseline renal function (eGFR), left ventricular ejection fraction (LVEF), anaemia, , proteinuria and preoperative IABP implantation. The information recorded during the operation included the presence of left main disease, [?]3 coronary artery lesions, [?]3 coronary anastomoses, operation time, on-pump CABG, and emergency surgery. Postoperative information included the use of blood products, ventilator time, ICU stay time. Related information about surgical complications (death, reoperation, perioperative myocardial infarction, respiratory complications, atrial fibrillation, stroke) was also included.

\section{Outcome Measures}

First, single-factor analysis was used to determine the factors with ap value less than 0.02 or that were clinically closely related to AKI. Then, multivariate logistic regression analysis was used to screen out the factors with $p<0.05$. Finally, At the same time, the incidence of complications after CABG was compared between the two groups.

\section{Statistical Analysis:}

SPSS 22.0 for Mac (IBM SPSS Statistics) was used for statistical analysis. Continuous variables were reported as the mean +/- standard deviation or median (interquartile range) (IQR). Categorical variables were reported as the absolute frequency and as a percentage. Student'st -test was applied for continuous data with equal or unequal variances. The Mann-Whitney U test was applied for continuous data that were not normally distributed. Pearson's $\chi^{2}$ and Fisher's exact tests were used for categorical data. All meaningful variables were included in a multiple logistic regression analysis. Logistic regression analysis was repeated using variables that were significant in the previous analysis ${ }^{15}$. Accuracy was measured by the area under the receiver operating characteristic (ROC) curve analysis. $p<0.05$ was considered to be statistically significant.

\section{Results:}

The baseline clinical data on age, sex, body mass index, smoking, left ventricular ejection fraction, and so on are shown in Table 1 . The incidence of AKI was $27.5 \%$. 
According to the results of univariate analysis and clinical significance, the following factors were included in the regression analysis: age, hypertension, smoking, eGFR, LVEF, anaemia, proteinuria, left main disease, [?]3 coronary artery lesions, [?]3 coronary anastomoses, preoperative IABP implantation, blood transfusion, and ventilator time (Table 2 ).

The results showed that eGFR, $(\mathrm{OR}=0.972,95 \% \mathrm{CI}=0.958-0.987, p<0.001)$, [?] 3 coronary anastomoses $(\mathrm{OR}=0.400,95 \% \mathrm{CI}=0.230-0.693, p=0.001)$, preoperative IABP implantation $(\mathrm{OR}=1.92,95 \% \mathrm{CI}=1.006$ $3.664, p=0.048)$ and ventilation time $(\mathrm{OR}=1.016,95 \% \mathrm{CI}=1.007-1.026, p=0.001)$ were independent risk factors for AKI. The results of multivariate regression analysis of these risk factors are shown in Table 3. The area under the ROC curve was 0.702, (95\% CI [0.643-0.761], $p<0.001)$ (Figure 1).

Postoperative complications indicated that 7 patients died in the AKI group, which was significantly higher than the number of deaths in the non-AKI group (7 (5.9\%) vs $0(0 \%) p<0.001)$. In addition, the rates of reoperation $(10(8.4 \%)$ vs $4(1.3 \%) p=0.001)$ and postoperative myocardial infarction $(34(28.6)$ vs $26(8.3 \%)$ $p<0.001$ ), were higher in the AKI group than in the non-AKI group. There was no significant difference in other complications between the two groups, as shown in Table 4.

\section{Discussion:}

Our results suggest that eGFR, [?]3 coronary anastomoses, preoperative IABP implantation and prolonged ventilation time are independent risk factors for AKI in elderly patients undergoing CABG.

The incidence of AKI was $27.5 \%$ in our study which is similar to the value reported in previous studies. The incidence of AKI in elderly patients was lower than that in Wilko Reents'study ${ }^{19}$. On the one hand, the reason may be that $40 \%$ of the patients had renal insufficiency before the operation in the Wilko Reents' Study. On the other hand, cardiopulmonary bypass surgery is closely related to acute kidney injury ${ }^{14}$, and only $17.1 \%(74 / 432)$ of the patients in this study received coronary artery bypass grafting under cardiopulmonary bypass, while more than half of the patients in the Wilko Reents'study received cardiopulmonary bypass surgery.

Previous studies have shown that preoperative anaemia and proteinuria were also independent risk factors for AKI after surgery ${ }^{20,21}$. According to the latest literature, these indicators were included in our study, but the final results were not consistent with those of previous studies. On the one hand, the results from previous studies may not be representative of elderly patients; on the other hand, the sample size of our study was insufficient.

eGFR was a risk factor for AKI in this study, which is consistent with many previous studies. Preoperative IABP implantation is an independent risk factor, which indicates that the use of IABP in elderly patients has greater impact on renal function. This is consistent with previous studies ${ }^{22-24}$. It is suggested that early operation after IABP implantation may reduce the incidence of AKI. In addition, the revascularization of more than 3 coronary anastomoses is a protective factor for AKI, which suggests that complete revascularization can not only benefit the heart but also benefit the renal function. A prolonged ventilator time can increase the incidence of AKI, which suggests that the occurrence of AKI can be reduced in patients if they can recover spontaneous breathing as soon as possible. This suggests that preoperative pulmonary function exercise is quite important.

In terms of postoperative complications, the mortality, postoperative myocardial infarction and reoperation rates in the AKI group were higher than those in the non-AKI group. Previous studies showed that acute kidney injury after coronary artery bypass grafting was associated with a long-term risk of myocardial infarction. Three percent to seven percent of patients undergoing CABG experienced myocardial infarction within one year after surgery ${ }^{25}$. Therefore, our study also suggests that AKI may be closely related to the occurrence of myocardial infarction and may even lead to postoperative death.

The limitations of this study were as follows: on the one hand, this study was a single-center, retrospective study, with certain selection bias; on the other hand, the sample size calculation suggested that the size was small and a larger sample needs to be included in future analyses. Finally, in this study, the diagnosis of 
AKI was based on KDIGO criteria. Because diuretics are used in many patients after surgery, urine volume was not used as one of the diagnostic criteria of AKI.

\section{Conclusions}

The eGFR, [?]3 coronary anastomoses, preoperative IABP implantation and prolonged ventilation time are independent risk factors for AKI in elderly patients undergoing coronary artery bypass grafting. Early intervention is important.

\section{Abbreviations}

CABG: Coronary artery bypass grafting; AKI: Acute kidney injury; KDIGO:

Kidney Disease Improving Global Outcomes; ROC: Receiver operating characteristic; eGFR: Estimated glomerular filtration rate; IABP: Intra-aortic balloon pump; CI: Confidence interval; ICU: Intensive care unit; BMI: Body mass index; NYHA: New York Heart Association; PCI: Percutaneous coronary intervention; LVEF: Left ventricular ejection fraction; IQR: Interquartile range; OR: Odds ratio.

\section{Figure legends and table legends}

Fig. 1 ROC curves using eGFR, [?]3 coronary anastomoses, IABP implantation, and ventilation time for predicting postoperative AKI

Table 1: AKI: Acute kidney injury; BMI: Body mass index; COPD: Chronic obstructive pulmonary diseases; MI: Myocardial infarction; PCI: Percutaneous coronary intervention; LVEF: Left ventricular ejection fraction; IQR: Interquartile range; eGFR: Estimated glomerular filtration rate; CABG: Coronary artery bypass grafting; IABP: Intra-aortic balloon pump; ICU: Intensive care unit

Table 2: AKI: Acute kidney injury; CABG: Coronary artery bypass grafting; OR: Odds ratio; eGFR: Estimated glomerular filtration rate; LVEF: Left ventricular ejection fraction; IABP: Intra-aortic balloon pump

Table 3 AKI: Acute kidney injury; CABG: Coronary artery bypass grafting; OR: Odds ratio; eGFR: Estimated glomerular filtration rate

Table 4 AKI: Acute kidney injury

\section{Authors' contributions}

Yang Li and Ran Dong were responsible for the design, supervision of the study, and revision of the manuscript. Xuejian Hou drafted the manuscript. Shijun Xu and Zhuhui Huang designed a statistical plan. Yang Li and Taoshuai Liu participated in the revision of the manuscript and the coordination of the study. Xuejian Hou and Zhuhui Huang participated in data acquisition. All authors read and agreed to the final manuscript.

\section{References}

1. Rosner MH, Okusa MD. Acute kidney injury associated with cardiac surgery. Clin J Am Soc Nephrol. 2006;1(1):19-32.

2. Dasta JF, Kane-Gill SL, Durtschi AJ, Pathak DS, Kellum JA. Costs and outcomes of acute kidney injury (AKI) following cardiac surgery.Nephrol Dial Transplant. 2008;23(6):1970-1974.

3. Brown JR, Kramer RS, Coca SG, Parikh CR. Duration of acute kidney injury impacts long-term survival after cardiac surgery. Ann Thorac Surg. 2010;90(4):1142-1148.

4. Hobson CE, Yavas S, Segal MS, et al. Acute kidney injury is associated with increased long-term mortality after cardiothoracic surgery. Circulation. 2009;119(18):2444-2453.

5. Corredor C, Thomson R, Al-Subaie N. Long-Term Consequences of Acute Kidney Injury After Cardiac Surgery: A Systematic Review and Meta-Analysis. J Cardiothorac Vasc Anesth. 2016;30(1):69-75. 
6. Thakar CV, Worley S, Arrigain S, Yared JP, Paganini EP. Improved survival in acute kidney injury after cardiac surgery. Am J Kidney Dis. 2007;50(5):703-711.

7. Lassnigg A, Schmidlin D, Mouhieddine M, et al. Minimal changes of serum creatinine predict prognosis in patients after cardiothoracic surgery: a prospective cohort study. J Am Soc Nephrol.2004;15(6):1597-1605.

8. Goldberg R, Dennen P. Long-term outcomes of acute kidney injury.Adv Chronic Kidney Dis. 2008;15(3):297-307.

9. Doyle JF, Forni LG. Acute kidney injury: short-term and long-term effects. Crit Care. 2016;20(1):188.

10. Mao MA, Thongprayoon C, Wu Y, et al. Incidence, Severity, and Outcomes of Acute Kidney Injury in Octogenarians following Heart Valve Replacement Surgery. Int J Nephrol. 2015;2015:237951.

11. Schrier RW, Wang W, Poole B, Mitra A. Acute renal failure: definitions, diagnosis, pathogenesis, and therapy. Journal of Clinical Investigation. 2004;114(1):5-14.

12. Bellomo R, Kellum JA, Ronco C. Acute kidney injury. The Lancet. 2012;380(9843):756-766.

13. Chen SW, Chang CH, Fan PC, et al. Comparison of contemporary preoperative risk models at predicting acute kidney injury after isolated coronary artery bypass grafting: a retrospective cohort study.BMJ Open. 2016;6(6):e010176.

14. Li Z, Fan G, Zheng X, et al. Risk factors and clinical significance of acute kidney injury after on-pump or off-pump coronary artery bypass grafting: a propensity score-matched study. Interact Cardiovasc Thorac Surg. 2019;28(6):893-899.

15. Ortega-Loubon C, Fernandez-Molina M, Paneda-Delgado L, Jorge-Monjas P, Carrascal Y. Predictors of Postoperative Acute Kidney Injury after Coronary Artery Bypass Graft Surgery. Braz J Cardiovasc Surg.2018;33(4):323-329.

16. Amini S, Najafi MN, Karrari SP, et al. Risk Factors and Outcome of Acute Kidney Injury after Isolated CABG Surgery: a Prospective Cohort Study. Braz J Cardiovasc Surg. 2019;34(1):70-75.

17. Yue Z, Yan-Meng G, Ji-Zhuang L. Prediction model for acute kidney injury after coronary artery bypass grafting: a retrospective study.Int Urol Nephrol. 2019;51(9):1605-1611.

18. Khwaja A. KDIGO clinical practice guidelines for acute kidney injury. Nephron Clin Pract. 2012;120(4):c179-184.

19. Reents W, Hilker M, Borgermann J, et al. Acute kidney injury after on-pump or off-pump coronary artery bypass grafting in elderly patients. Ann Thorac Surg. 2014;98(1):9-14; discussion 14-15.

20. Oprea AD, Del Rio JM, Cooter M, et al. Pre- and postoperative anemia, acute kidney injury, and mortality after coronary artery bypass grafting surgery: a retrospective observational study. Can J Anaesth. 2018;65(1):46-59.

21. Kwon JT, Jung TE, Lee DH. Predictive risk factors of acute kidney injury after on-pump coronary artery bypass grafting. Ann Transl Med. 2019;7(3):44.

22. Brown JR, Cochran RP, Leavitt BJ, et al. Multivariable Prediction of Renal Insufficiency Developing After Cardiac Surgery. Circulation. 2007;116(11_suppl):I-139-I-143.

23. Nah CW, Ti LK, Liu W, Ng RR, Shen L, Chew ST. A clinical score to predict acute kidney injury after cardiac surgery in a Southeast-Asian population. Interact Cardiovasc Thorac Surg. 2016;23(5):757-761.

24. Karkouti K, Wijeysundera DN, Yau TM, et al. Acute kidney injury after cardiac surgery: focus on modifiable risk factors. Circulation. 2009;119(4):495-502. 
25. Javaid A, Steinberg DH, Buch AN, et al. Outcomes of Coronary Artery Bypass Grafting Versus Percutaneous Coronary Intervention With Drug-Eluting Stents for Patients With Multivessel Coronary Artery Disease. Circulation. 2007;116(11_suppl):I-200-I-206.

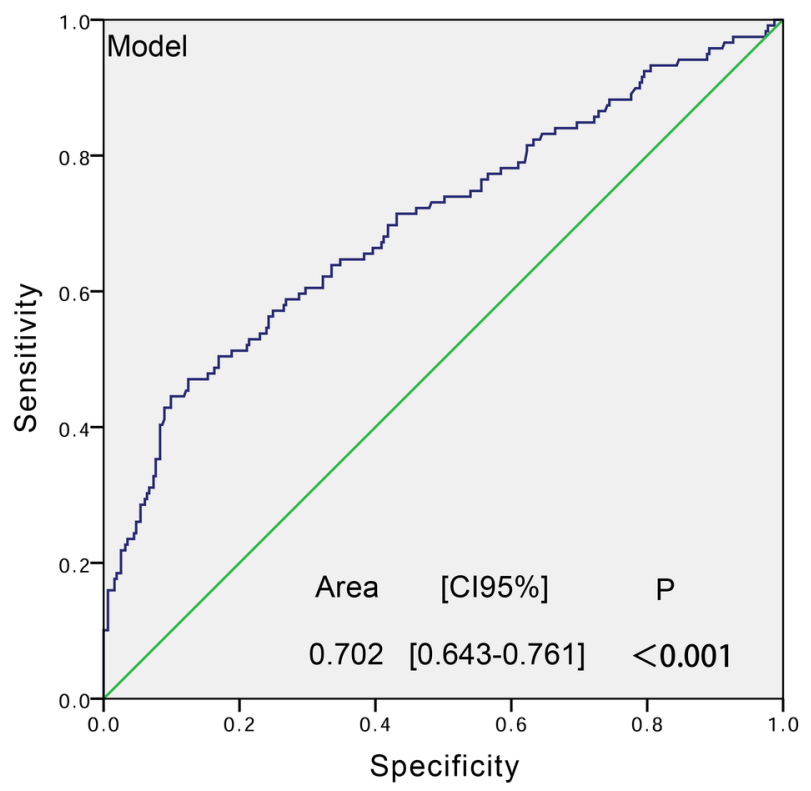

Fig. 1 ROC curves using eGFR, $\geq 3$ coronary anastomoses, IABP implantation, ventilation time for predicting postoperative AKI

\section{Hosted file}

Table 1.pdf available at https://authorea.com/users/397325/articles/510240-risk-factors-foracute-kidney-injury-in-elderly-patients-after-coronary-artery-bypass-grafting-a-chinesepopulation

\section{Hosted file}

Table 2.pdf available at https://authorea.com/users/397325/articles/510240-risk-factors-foracute-kidney-injury-in-elderly-patients-after-coronary-artery-bypass-grafting-a-chinesepopulation

\section{Hosted file}

Table 3.pdf available at https://authorea.com/users/397325/articles/510240-risk-factors-foracute-kidney-injury-in-elderly-patients-after-coronary-artery-bypass-grafting-a-chinesepopulation

\section{Hosted file}

Table 4.pdf available at https://authorea.com/users/397325/articles/510240-risk-factors-foracute-kidney-injury-in-elderly-patients-after-coronary-artery-bypass-grafting-a-chinesepopulation 